\title{
Электронные состояния доноров V группы в германии: вариационный расчет с учетом короткодействующего потенциала
}

\author{
(C) А.А. Ревин ${ }^{1,2}$, А.М. Михайлова ${ }^{1,2}$, А.А. Конаков ${ }^{1,2, \uparrow}$, В.Н. Шастин ${ }^{2}$ \\ ${ }^{1}$ Национальный исследовательский Нижегородский государственный университет им. Н.И. Лобачевского, \\ 603950 Нижний Новгород, Россия \\ ${ }^{2}$ Институт ффизики микроструктур Российской академии наук, \\ 603087 Нижний Новгород, Россия \\ `E-mail: konakov_anton@mail.ru
}

Поступила в Редакцию 15 апреля 2020 г.

В окончательной редакции 21 апреля 2020 г.

Принята к публикации 21 апреля 2020 г.

В рамках приближения огибающей функции рассчитаны волновые функции низколежащих $1 s\left(A_{1}\right)-, 2 s-$, $2 p_{0^{-}}, 2 p_{ \pm-}, 3 p_{0^{-}}$состояний мелких донорных центров $\mathrm{P}, \mathrm{As}, \mathrm{Sb}$ в германии с учетом короткодействующего потенциала примеси. Последний построен индивидуально для каждой примеси с учетом пространственной дисперсии диэлектрической проницаемости и различия между ионными остовами германия и примесного центра. Уравнение для огибающей решалось с использованием вариационного метода Ритца, при этом выбранные пробные функции орбитально-невырожденных $s$-состояний характеризуются двумя пространственными масштабами: первый, порядка эффективного боровского радиуса донора, отвечающий дальнодействующей части потенциала, и второй, на порядок меньший, моделирующий отклик электрона на короткодействующую часть потенциала донора. Электронная плотность в основном состоянии доноров смещается к ядру, что обусловлено учетом притягивающего потенциала „центральной ячейки“. Огибающие функции р-состояний в свою очередь построены так, что являются ортогональными огибающим в основном состоянии для каждого примесного центра, и в отличие от предыдущих работ различны для разных доноров.

Ключевые слова: германий, мелкие доноры, короткодействующий потенциал, приближение огибающей функции.

DOI: 10.21883/FTP.2020.09.49836.30

\section{1. Введение}

Экспериментальное и теоретическое исследование электронных свойств доноров V группы в ковалентных полупроводниках (в первую очередь кремнии и германии) ведется еще с 50-х годов ХХ века $[1,2]$. Эти объекты вызывают значительный интерес и сейчас в связи с возможным их приложением в области построения квантовых компьютеров [3,4], источников когерентного ТГц излучения [5,6] и „одноатомных“ транзисторов [7]. Традиционно для различных приложений более активно изучаются электронные свойства мелких примесных центров в кремнии, что обусловлено широкой распространенностью материала в природе, возможностями его глубокой химической и изотопной очистки и управления концентрацией примесных центров, а также ориентацией на технологическую базу электроники. В то же время слабо легированный германий $n$-типа обладает рядом преимуществ. Во-первых, пространственный масштаб волновых функций основного состояния локализованных на донорах электронов оказывается там в $~ 4$ раза больше, чем в кремнии [8], что заметно расширяет возможности управления состояниями посредством приложения внешнего электрического поля [9]. Вместе с увеличением масштаба волновых функций в германии по сравнению с кремнием уменьшаются энергии возбуждения и ионизации мелких доноров [8], что позволяет, например, получать более длинноволновое излучение, необходимое для решения ряда прикладных задач [10]. Кроме того, в приложениях спинтроники и квантовых вычислений существенно спин-орбитальное взаимодействие, которое в германии значительно сильнее, чем в кремнии. Вместе с развитием эпитаксиальной технологии германия на кремнии [11] указанные преимущества делают изучение электронных и оптических свойств доноров Vгруппы в германии весьма актуальным и с фундаментальной, и с прикладной точек зрения.

Энергии электронных состояний доноров Vгруппы в германии определены экспериментально методами ИК-спектроскопии [12] и фотопроводимости [13]. Результаты проведенных в $[12,13]$ измерений энергий орбитально-вырожденных возбужденных состояний $(2 p$ и выше) находятся в хорошем согласии с теоретическими расчетами Фалкнера [14] в рамках метода эффективной массы, тогда как основное $1 s$-состояние расщепляется на синглет $1 s\left(A_{1}\right)$ и триплет $1 s\left(T_{2}\right)[15]$ вследствие долинно-орбитального взаимодействия [16], обусловленного короткодействующей частью примесного потенциала (коррекцией „центральной ячейки“) [17].

Однако информации об энергетическом спектре в ряде случаев оказывается недостаточно. В частности, при анализе темпов излучательных и безызлучательных переходов между электронными состояниями доноров $[18,19]$ необходимо знание о волновых функциях локализован- 
ных носителей, которые в отличие от измеряемых экспериментально энергий требуется рассчитывать. Кроме того, волновые функции локализованных на донорах электронов необходимы для анализа сверхтонкого взаимодействия со спином ядра [20] и определения величины эффекта Штарка [21].

Ввиду большого пространственного масштаба волновых функций электронов, локализованных на мелких донорах в германии ( $\sim 10$ нм в основном состоянии $[14,20])$, приближение огибающей функции становится наиболее оптимальным с точки зрения соотношения точности и вычислительной „стоимости“ расчета. Ранее метод огибающей функции был применен к решению спектральной задачи для доноров в германии в работах $[14,20,22]$. В работах $[14,22]$ рассчитывались как функции основного состояния, так и возбужденных, однако не принималась во внимание короткодействующая часть потенциала донора, наиболее существенная в основном состоянии. В то же время в [20] были получены функции состояний $1 s\left(A_{1}\right)$ и $1 s\left(T_{2}\right)$ для электронов, локализованных на донорах $\mathrm{P}$, As, Bi с учетом короткодействующей части потенциала. Однако эти функции не тестировались на предмет ортогональности функциям возбужденных состояний.

В настоящей работе с применением приближения огибающей функции и вариационного метода Ритца рассчитаны волновые функции низколежащих $1 s\left(A_{1}\right), 2 s$, $2 p_{0}, 2 p_{ \pm}, 3 p_{0}$-состояний мелких донорных центров $\mathrm{P}$, $\mathrm{As}, \mathrm{Sb}$ в германии с учетом короткодействующего потенциала примеси. Выбор именно этих состояний для расчета обусловлен тем, что они являются рабочими при создании инверсии населенностей примесных уровней в $n$-Ge $[18,19]$. Короткодействующая часть потенциала построена индивидуально для каждой примеси, что позволяет различить волновые функции как основного, так и возбужденных состояний для каждой примеси.

\section{2. Гамильтониан задачи}

Мы стартуем с истинного гамильтониана $\hat{H}(\mathbf{r})$ электрона, локализованного на доноре замещения V группы в германии, который имеет вид

$$
\hat{H}(\mathbf{r})=\frac{\hat{\mathbf{p}}^{2}}{2 m_{0}}+V_{0}(\mathbf{r})+U(\mathbf{r}),
$$

где $m_{0}$ - масса свободного электрона, $V_{0}(\mathbf{r})-$ периодический потенциал кристаллической структуры, а $U(\mathbf{r})-$ потенциал примесного центра, и определяется главным образом тремя эффектами: различием в ионных остовах донора и германия, деформацией ближайшего окружения, вносимого донором, а также дисперсией диэлектрической проницаемости среды [17]. В силу того что нас будут интересовать состояния вблизи дна орбитальновырожденной зоны проводимости, в (1) опущено спинорбитальное взаимодействие наравне с другими релятивистскими поправками. Поскольку ширина запрещенной зоны германия при гелиевой температуре $\sim 0.74$ эВ [23], а известные из эксперимента энергии ионизации мелких доноров составляют $\sim 0.012$ эВ [8], волновые функции $\Psi(\mathbf{r})$ локализованных состояний определяются блоховскими функциями вблизи дна зоны проводимости и в рамках приближения огибающей могут быть записаны в виде

$$
\Psi(\mathbf{r})=\sum_{j=1}^{4} \psi_{j}(\mathbf{r}) \varphi_{j}(\mathbf{r})
$$

где $\varphi_{j}(\mathbf{r})$ - блоховские функции состояний четырех энергетических минимумов долинно-вырожденного дна зоны проводимости германия, $\psi_{j}(\mathbf{r})$ - отвечающие им компоненты огибающей.

С использованием (2) на основе гамильтониана (1) может быть получено уравнение для огибающих $\psi_{j}(\mathbf{r})$ в рамках многодолинного приближения эффективной массы $[17,20]$. Однако мы a posteriori будем работать в однодолинном приближении. Во-первых, для состояний выше $1 s$ оно обосновано с высокой точностью [17,24]. Во-вторых, для доноров Vгруппы энергии состояния $1 s\left(T_{2}\right)$ мало отличаются от предсказываемых в рамках однодолинной модели с водородоподобным потенциалом $U(\mathbf{r})[8]$, в то время как уровень $1 s\left(A_{1}\right)$ существенно отщепляется вниз. Поскольку вклад всех долин в синглетное состояние равный, химический сдвиг уровня $1 s\left(A_{1}\right)$ будем рассматривать в однодолинном приближении. В результате для отыскания волновых функций искомых состояний в приближении огибающей однодолинное уравнение шредингеровского типа примет вид [17]

$$
\left(\frac{1}{2 m_{\perp}}\left(\hat{p}_{x}^{2}+\hat{p}_{y}^{2}+\gamma \hat{p}_{z}^{2}\right)+U(\mathbf{r})\right) \psi(\mathbf{r})=E \psi(\mathbf{r}),
$$

где $\gamma=m_{\perp} / m_{\|}-$параметр анизотропии гамильтониана, $m_{\|}=1.588 m_{0}$ и $m_{\perp}=0.0815 m_{0}[25]-$ продольная и поперечная эффективные массы вблизи дна зоны проводимости германия соответственно, вдоль оси долины ориентирована ось $z$ декартовой системы координат, а $E-$ рассчитываемая энергия квантового состояния. Заметим, что в (3) вследствие использования однодолинного приближения мы опустили нижний индекс у огибающей функции $\psi(\mathbf{r})$.

Потенциал $U(\mathbf{r})$ может быть задан фурье-образом $\tilde{U}(\mathbf{q})$,затравочного“ потенциала как

$$
U(\mathbf{r})=\frac{1}{(2 \pi)^{3}} \int d \mathbf{q} \varepsilon^{-1}(\mathbf{q}) \tilde{U}(\mathbf{q})
$$

где $\varepsilon(\mathbf{q})$ - статическая диэлектрическая проницаемость среды, в длинноволновом пределе равная $\varepsilon_{s}=16.0$ [26], в коротковолновом - единице, и являющаяся квадратичной функцией волнового вектора при малых q [27]. Пренебрегая анизотропией диэлектрической функции, $\varepsilon^{-1}(\mathbf{q})$ может быть записана с использованием следую- 
щего аппроксиманта [28,29]:

$$
\varepsilon^{-1}(\mathbf{q})=\frac{1}{\varepsilon_{s}}+\frac{B_{0}|\mathbf{q}|^{2}}{\eta_{0}^{2}+|\mathbf{q}|^{2}}+\left(1-B_{0}-\frac{1}{\varepsilon_{s}}\right) \frac{|\mathbf{q}|^{2}}{\kappa_{0}^{2}+|\mathbf{q}|^{2}},
$$

где параметры $B_{0}, \eta_{0}$ и $\kappa_{0}$ подбираются таким образом, чтобы максимально точно соответствовать результатам численных расчетов диэлектрической функции. Использование трехпараметрической функции позволяет достаточно точно описать расчетные зависимости диэлектрической функции не только при малых и больших значениях волновых векторов, но и в промежуточной области. Если использовать диэлектрическую функцию, приведенную в работе [26], то параметры аппроксиманта (5) будут $B_{0}=0.78, \eta_{o}=1.26 a_{0}^{-1}$ и $\kappa_{0}=1.17 a_{0}^{-1}$, где $a_{0}=0.0529$ нм - истинный боровский радиус.

Наибольшая трудность при вычислении потенциала $U(\mathbf{r})$ связана с определением фурье-образа $\tilde{U}(\mathbf{q})$ „затравочного“ потенциала. Одним из путей к его расчету является применение псевдопримесного подхода, разработанного Пантелидесом и Са [30]. Недостатком этого метода является отсутствие учета локальных деформаций, вызванных различием в ковалентных радиусах германия и примеси, а также неоднозначность в определении псевдопотенциалов. Вместо этого мы применим следующий подход. Рассчитаем сначала потенциал (4) с диэлектрической функцией (5) и фурьеобразом $\tilde{U}_{\text {p.c. }}(\mathbf{q})=4 \pi e_{0}^{2} / \mathbf{q}^{2}$ потенциала единичного точечного заряда, расположенного в начале координат:

$$
\begin{aligned}
& U_{p . c .}(\mathbf{r})=-\frac{e_{0}^{2}}{\varepsilon_{s}|\mathbf{r}|}-\frac{e_{0}^{2}}{|\mathbf{r}|} \\
& \times\left(B_{0} \exp \left(-\eta_{0}|\mathbf{r}|\right)+\left(1-B_{0}-\frac{1}{\varepsilon_{s}}\right) \exp \left(-\kappa_{0}|\mathbf{r}|\right)\right),
\end{aligned}
$$

где $e_{0}-$ элементарный заряд. Отметим, что изотропия потенциала (4) и отсутствие тетраэдрической симметрии, обусловленной окружением центра, связаны с пренебрежением анизотропией в функции (3).

Первое слагаемое в (6) есть водородоподобный дальнодействующий потенциал примеси, одинаковый для любого донора замещения V группы, а второе слагаемое короткодействующая часть потенциала, существенная на масштабах элементарной ячейки. Именно она и чувствительна к типу донора и определяет химический сдвиг уровня $1 s\left(A_{1}\right)$. Однако химическая природа донора никак не учтена в (6), поскольку потенциал строился для единичного точечного заряда. В случае же примесного центра на малых масштабах экранируется не единичный точечный заряд, а распределенная в пространстве плотность, что в действительности может существенно менять короткодействующую часть потенциала. Поэтому модифицируем выражение (6) следующим образом: будем считать, что его функциональная связь с r остается прежней, а параметры $B_{0}, \eta_{0}$ и $\kappa_{0}$ заменяются на параметры $B, \eta$ и $\kappa$, подбираемые индивидуально для каждого донора. Также нами вносится дополнительный безразмерный множитель $Q$ перед короткодействующим потенциалом (величина $Q e_{0}$ играет роль эффективного заряда, экранируемого на малом масштабе от ядра). В результате потенциал донора $U(\mathbf{r})$ принимает вид

$$
\begin{aligned}
& U(\mathbf{r})=-\frac{e_{0}^{2}}{\varepsilon_{s}|\mathbf{r}|}-\frac{Q e_{0}^{2}}{|\mathbf{r}|} \\
& \times\left(B \exp (-\eta|\mathbf{r}|)+\left(1-B-\frac{1}{\varepsilon_{s}}\right) \exp (-\kappa|\mathbf{r}|)\right),
\end{aligned}
$$

где параметры $Q, B, \eta$ и $\kappa$ подбираются таким образом, чтобы наиболее близко отвечать экспериментальным данным по энергии основного состояния донора.

\section{3. Метод решения}

Уравнение (1) для огибающей с потенциалом (7) решалось вариационным методом Ритца. При решении мы отдельно выделили сферически симметричные состояния $\left(1 s\left(A_{1}\right)\right.$ и $\left.2 s\right)$ и орбитально-вырожденные состояния $p$-типа. При расчете волновой функции состояния $1 s$ в модели с анизотропной эффективной массой традиционно используется двухпараметрическая пробная функция вида $[14,24]$

$$
\psi_{1 s}(\mathbf{r})=N_{0} \exp \left(-\sqrt{x^{2}+y^{2}+(\beta / \gamma) z^{2}} / R\right)
$$

где вариационные параметры $R$ и $\beta$ характеризуют эффективный масштаб и анизотропию огибающей основного состояния, а $N_{0}$ - действительный нормировочный множитель, удовлетворяющий условию $\int d \mathbf{r}|\psi(\mathbf{r})|^{2}=1$. Функция (8) хорошо отвечает водородоподобной модели, однако не описывает химический сдвиг, обусловленный потенциалом „центральной ячейки“.

Для расчета энергии и огибающей функции основного состояния $1 s\left(A_{1}\right)$ мелкого донора в германии мы использовали четырехпараметрическую пробную функцию вида

$$
\begin{aligned}
\psi_{1 s}(\mathbf{r})= & N_{1}\left(\exp \left(-\sqrt{x^{2}+y^{2}+(\beta / \gamma) z^{2}} / R_{1}\right)\right. \\
& \left.+C_{1} \exp \left(-\sqrt{x^{2}+y^{2}+(\beta / \gamma) z^{2}} / R_{2}\right)\right)
\end{aligned}
$$

где $R_{1}$ и $R_{2}$ имеют смысл эффективных радиусов волновой функции, один из которых по смыслу отражает реакцию на дальнодействующую составляющую потенциала, а второй - на короткодействующую, $C_{1}-$ безразмерный „весовой“ множитель, определяющий „смесь““ этих вкладов, $N_{1}-$ нормировочный множитель, а $\beta$ имеет тот же смысл, что и в (8). 
Для расчета состояния $2 s$ использовалась функция вида

$$
\begin{aligned}
\psi_{2 s}(\mathbf{r})= & N_{2}\left(\exp \left(-\sqrt{x^{2}+y^{2}+(\beta / \gamma) z^{2}} / R_{3}\right)\right. \\
& \left.+C_{2} \exp \left(-\sqrt{x^{2}+y^{2}+(\beta / \gamma) z^{2}} / R_{4}\right)\right) \\
& \times\left(1+\chi_{2} \sqrt{x^{2}+y^{2}+(\beta / \gamma) z^{2}}\right)
\end{aligned}
$$

где $R_{3}, R_{4}, C_{2}$ и $N_{2}$ имеют тот же смысл, что $R_{1}, R_{2}, C_{1}$ и $N_{1}$ в (9), $\chi_{2}$ - параметр, обеспечивающий ортогональность основному состоянию, а значение $\beta$ берется то же, что и для основного состояния в (9). Состояние $2 s$ необходимо описывать пробной функцией с двумя масштабами ввиду той же симметрии, что и состояние $1 s\left(A_{1}\right)$.

Возбужденные состояния $p$-типа моделировались функциями, предложенными в [14]:

$$
\left.\psi_{n l m}(\mathbf{r})=(\beta / \gamma)^{1 / 4} f_{n l m}\left(x, y,(\beta / \gamma)^{1 / 2}\right) z\right),
$$

где $f_{n l m}(\mathbf{r})$ - волновые функции атома водорода с набором квантовых чисел $n l m$. Они содержат тот же параметр $\beta$, что (9) и (10), что гарантирует ортогональность функций. В случае состояний $p$-типа достаточно выбрать пробные функции с единственным пространственным масштабом, поскольку ортогональность функций $s$-состояний достигается за счет анизотропии функций (11).

\section{4. Результаты расчетов и их обсуждение}

В ходе вариационной процедуры отыскания функции (9) нами найдены параметры огибающих функций, наиболее близко отвечающие имеющимся экспериментальным данным по энергии ионизации мелких доноров в германии [8]. Результаты вариационных расчетов оказались сильно чувствительны к параметрам $\eta$ и $\kappa$ короткодействующего потенциала (7), характеризующих его пространственный масштаб, и в заметно меньшей степени зависимы от значений $B$ и $Q$, фактически задающих его мощность. При этом для каждого донора подбирался свой набор характеристик короткодействующего потенциала, определяющий в итоге и отличие между энергиями локализованных электронов. В качестве нулевого приближения для параметров короткодействующего потенциала использовались значения $B_{0}, \eta_{0}, \kappa_{0}$ и $Q_{0}=1$, точно соответствующие потенциалу (6) экранированного точечного заряда. Далее эти параметры модифицировались для каждого донора, чтобы получить наилучшее согласие с измеренными энергиями основного состояния. Полученные таким образом характеристики короткодействующего потенциала приведены в табл. 1. В свою очередь на рис. 1 графически представлены зависимости короткодействующих
Таблица 1. Характеристики короткодействующего потенциала доноров замещения V группы в германии

\begin{tabular}{l|c|c|c|c}
\hline Донор & $B$ & $\eta\left(a_{0}^{1}\right)$ & $\kappa\left(a_{0}^{-1}\right)$ & \multicolumn{1}{|c}{$Q$} \\
\hline $\mathrm{Ge}: \mathrm{P}$ & 3.4 & 0.83 & 1.62 & 1.27 \\
$\mathrm{Ge}: \mathrm{As}$ & 3.3 & 0.82 & 1.64 & 1.324 \\
$\mathrm{Ge}: \mathrm{Sb}$ & 2.3 & 1.14 & 1.23 & 3.47
\end{tabular}

потенциалов для различных доноров в сравнении друг с другом и с экранированным в длинноволновом пределе кулоновским потенциалом.

Как видно из табл. 1 и рис. 1 короткодействующие потенциалы для фосфора и мышьяка в германии оказываются близки, но заметно отличаются от потенциала для сурьмы. В частности, существенное различие есть как в характеристиках масштаба, так и в величине $Q$. Отличие в параметрах $\eta$ и $\kappa$ может диктоваться существенно большим ковалентным радиусом сурьмы в сравнении с мышьяком и фосфором. Атом мышьяка является изокорным центром в германии и вносит минимальные искажения в ближайшее окружение. Атом фосфора легкий и обладает меньшим в сравнении с германием ковалентным радиусом, так что также не приводит к существенному изменению длин связей в тетраэдре окружающих его германиевых атомов. В то же время тяжелый донор сурьмы должен заметно деформировать ближайшее окружение (первую координационную сферу), приводя к локальному изменению плотности валентных электронов и вместе с этим к масштабу экранирования примесного центра. Параметр $Q$ в свою очередь является интегральной характеристикой экранируемого вблизи примесного ядра заряда. Заметно большее число остовных электронов атома сурьмы в сравнении с германием, мышьяком и фосфором в прин-

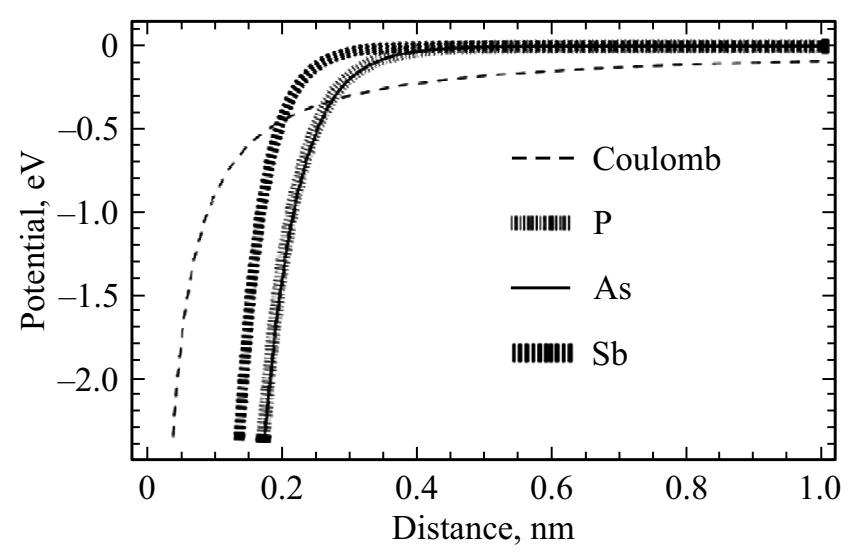

Рис. 1. Потенциальная энергия электрона, локализованного на мелкой примеси в $\mathrm{Ge}$, в зависимости от расстояния до примесного центра. Отдельно выделен дальнодействующий кулоновский вклад (штриховой линией) и короткодействующие вклады, относящиеся к различным примесям (P, As, $\mathrm{Sb})$. 
Таблица 2. Энергии и параметры огибающих функций основного $1 s\left(A_{1}\right)$-состояния электрона, локализованного на мелком доноре V группы в германии

\begin{tabular}{l|c|c|c|c|c|c|c|c|c}
\hline \multirow{2}{*}{ Донор } & \multirow{2}{*}{$\begin{array}{c}\text { Энергия } E, \\
\text { экспериментальные } \\
\text { данные (мэB) }[8]\end{array}$} & \multicolumn{3}{|c|}{$\begin{array}{c}\text { Расчет, } \\
\text { двухпараметрическая функция }\end{array}$} & \multicolumn{5}{|c}{ Расчет, } \\
\cline { 3 - 10 } & $E($ мэВ) & $R\left(a_{\mathrm{B}}\right)$ & $\beta$ & $E($ мэB) & $R_{1}\left(a_{\mathrm{B}}\right)$ & $R_{2}\left(a_{\mathrm{B}}\right)$ & $\beta$ & $C_{1}$ \\
\hline $\mathrm{Ge}: \mathrm{P}$ & -12.87 & -10.05 & 0.53 & 0.46 & -12.86 & 0.37 & 0.019 & 0.50 & 3.21 \\
$\mathrm{Ge}: \mathrm{As}$ & -14.18 & -10.09 & 0.53 & 0.46 & -14.21 & 0.32 & 0.019 & 0.50 & 3.53 \\
$\mathrm{Ge}: \mathrm{Sb}$ & -10.45 & -9.53 & 0.57 & 0.44 & -10.44 & 0.51 & 0.019 & 0.48 & 2.31
\end{tabular}

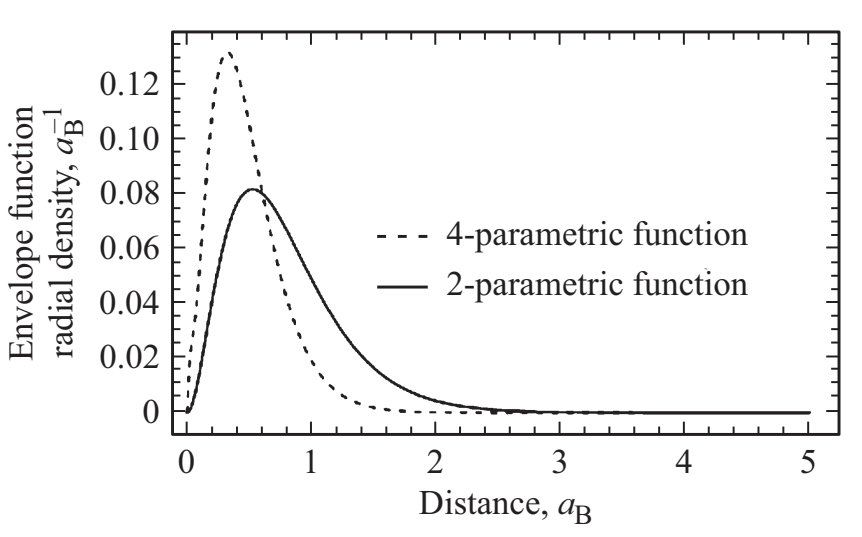

Рис. 2. Радиальная плотность огибающей функции для двух пробных функций основного $1 s\left(A_{1}\right)$ электронного состояния в системе $\mathrm{Ge}:$ As, рассчитанных с учетом короткодействующего потенциала, в зависимости от расстояния до примесного центра.

ципе соответствует тому, что на малом расстоянии от ядра сурьмы экранируется эффективно больший заряд.

Результаты расчета огибающей функции в основном состоянии с использованием пробной функции (9) в сравнении с экспериментальными данными и аналогичной процедурой, проведенной для традиционной двухпараметрической функции (8), приведены в табл. 2. Результаты измерения энергии приведены в мэВ, а масштаба волновой функции - в эффективных боровских радиусах донора $a_{\mathrm{B}}=\hbar^{2} \varepsilon_{s} / m_{\perp} e_{0}^{2} \approx 10.39 \mathrm{Hм}\left(a_{\mathrm{B}} \approx 18.36 a_{\mathrm{Ge}}\right.$, где $a_{\mathrm{Ge}}=0.566 \mathrm{Hм}$ - параметр решетки германия).

Как видно из результатов, представленных в табл. 2, один из радиусов $\left(R_{1}\right)$ оказывается существенно больше параметра решетки германия и фактически описывает поведение волновой функции на большом расстоянии от примесного центра, когда его уже можно считать водородоподобным. Второй $\left(R_{2}\right)$ - малый и определяется короткодействующим потенциалом примеси. Доля вклада с малым радиусом увеличивается, если основное состояние залегает глубже по энергии (в ряду центров $\mathrm{Ge}: \mathrm{Sb}, \mathrm{Ge}: \mathrm{P}, \mathrm{Ge}: \mathrm{As})$.

В качестве иллюстрации полученного результата на рис. 2 приведены радиальные плотности $|\psi(\mathbf{r})|^{2} r^{2}$ огибающей в основном состоянии Ge:As, посчитанные с каждой из пробных функций. Как видно, плотность, отвечающая функции, характеризуемой двумя радиусами, смещается ближе к положению примесного центра и возрастает. Эти изменения должны сказываться на матричных элементах переходов, а также на величине сверхтонкого взаимодействия электронного спина примеси и спина ядра.

На основе параметров, рассчитанных в основном состоянии можно найти энергии и параметры возбужденных состояний. Результаты расчета $2 s$-состояния приведены в табл. 3. За неимением экспериментальных данных для энергии состояния $2 s$ укажем для сравнения лишь значение, вычисленное с водородоподобным потенциалом и функцией (8), для которой значение $R$ помещено в колонку , $R_{3}{ }^{6 “}$.

В табл. 4 приведены результаты расчетов волновых функций для возбужденных $p$-состояний. В отличие от

Таблица 3. Результаты расчета энергий и волновых функций $2 s$-состояния с учетом короткодействующего потенциала и без его учета

\begin{tabular}{c|c|c|c|c|c}
\hline Донор & $E($ мэВ $)$ & $R_{3}\left(a_{\mathrm{B}}\right)$ & $R_{4}\left(a_{\mathrm{B}}\right)$ & $\beta$ & $C_{2}$ \\
\hline $\mathrm{Ge}: \mathrm{P}$ & -2.27 & 1.15 & 0.03 & 0.50 & 3.20 \\
$\mathrm{Ge}: \mathrm{As}$ & -2.38 & 1.14 & 0.09 & 0.50 & 0.51 \\
$\mathrm{Ge}: \mathrm{Sb}$ & -1.95 & 1.34 & 0.09 & 0.48 & 0.49 \\
$\mathrm{H}-$ like & -2.25 & 0.62 & - & 0.41 & -
\end{tabular}

Таблица 4. Результаты расчетов для возбужденных состояний $p$-типа в сравнении с известными экспериментальными данными. $R_{p}$ - масштаб (единственный) огибающей

\begin{tabular}{|c|c|c|c|c|}
\hline \multirow[b]{2}{*}{ Состояние } & \multirow[b]{2}{*}{ Донор } & \multirow{2}{*}{$\begin{array}{c}\text { Энергия } E, \\
\text { экспериментальные } \\
\text { данные (мэВ) [8] }\end{array}$} & \multicolumn{2}{|c|}{ Результаты расчетов } \\
\hline & & & $\begin{array}{c}\text { Энергия } E \\
\text { (мэВ) }\end{array}$ & $\begin{array}{c}R_{p} \\
\left(a_{\mathrm{B}}\right)\end{array}$ \\
\hline \multirow[t]{4}{*}{$2 p_{0}$} & Ge:P & -4.72 & -4.24 & 2.37 \\
\hline & $\mathrm{Ge}: \mathrm{As}$ & -4.74 & -4.24 & 2.37 \\
\hline & $\mathrm{Ge}: \mathrm{Sb}$ & -4.74 & -4.28 & 2.40 \\
\hline & $\mathrm{Ge}: \mathrm{P}$ & -2.54 & -1.91 & 2.91 \\
\hline \multirow[t]{3}{*}{$3 p_{0}$} & Ge:As & -2.57 & -1.91 & 2.91 \\
\hline & $\mathrm{Ge}: \mathrm{Sb}$ & -2.75 & -1.91 & 3.01 \\
\hline & Ge:P & -1.72 & & \\
\hline \multirow[t]{2}{*}{$2 p_{ \pm}$} & $\mathrm{Ge}: \mathrm{As}$ & -1.74 & -1.60 & 3.30 \\
\hline & $\mathrm{Ge}: \mathrm{Sb}$ & -1.71 & & \\
\hline
\end{tabular}


результатов, полученных ранее в [14], здесь параметр $\beta$ зависит от типа примеси, поскольку был получен при расчетах основного состояния электронов, локализованных на донорах с различным короткодействующим потенциалом.

Из табл. 4 видно, что значения энергий возбужденных $p$-состояний оказываются несколько выше, чем следует из эксперимента, однако возникает пусть и небольшая, но коррелирующая с экспериментальными данными зависимость от типа примеси, которая ранее никакими теоретическими расчетами не обосновывалась. Отметим, что в работе [14] значения энергии возбужденных состояний не зависели от типа примесного центра (задача решалась для водородоподобного потенциала), но были в целом ниже и лучше согласовывались с экспериментальными данными. Это, в частности, связано с тем, что в [14] при расчетах значение диэлектрической проницаемости германия было несколько занижено по сравнению с известным и составляло 15.4 вместо 16.0. Кроме того, различие может быть связано и с подбором короткодействующего потенциала для каждой примеси: он отыскивался так, чтобы наилучшим образом удовлетворять известным экспериментальным данным по энергиям основного состояния. По всей видимости, требуется более аккуратный поиск параметров, позволяющий описать и экспериментальные данные по возбужденным состояниям.

Обсудим теперь недостатки предложенного подхода и границы его применимости. Во-первых, конечно, изначально мы пренебрегали междолинным смешиванием, индуцированным короткодействующим потенциалом. Многодолинность волновой функции и долинноорбитальное расщепление могут быть существенны в ряде задач, интересных с точки зрения приложений (доноры в присутствии внешнего электрического поля, механических напряжений или вблизи поверхности германия). В этом случае предложенный нами подход должен быть модифицирован, например, в духе расчетов, представленных в [17,31].

Во-вторых, введенный короткодействующий потенциал (7) является сферически симметричным, тогда как истинный потенциал донора замещения должен, строго говоря, обладать тетраэдрической симметрией. Учет симметрии потенциала существен, согласно [32], при определении констант сверхтонкого взаимодействия. В принципе расчет такого потенциала в первом приближении может быть выполнен с помощью изложенного в работе алгоритма, но с использованием более точной анизотропной диэлектрической функции кристалла. В рамках предложенной модели учет анизотропии потенциала приведет к тетраэдрическому искажению огибающей в основном состоянии и некоторому понижению его энергии вследствие смешивания сферически симметричного $1 s\left(A_{1}\right)$-состояния с возбужденными $f$-состояниями [32]. Величина эффекта может быть оценена, например, по теории возмущений, однако количественный анализ выходит за рамки этой работы и может выступать предметом дальнейших исследований.

\section{5. Заключение}

В работе в рамках приближения огибающей функции разработан подход к вычислению энергий и волновых функций основного состояния мелких донорных центров $\mathrm{V}$ группы в германии. Для каждой примеси расчет проведен с учетом короткодействующего потенциала (коррекции „центральной ячейки“) в изотропном приближении, который подбирался, чтобы давать наилучшее согласие с результатами измерения энергий основного состояния в случае каждого донора. Вычислены огибающие волновых функций состояний $1 s\left(A_{1}\right), 2 s, 2 p_{0}$, $2 p_{ \pm}, 3 p_{0}$. Показано, что в основном $1 s\left(A_{1}\right)$-состоянии электронная плотность смещается к ядру как следствие учета короткодействующего потенциала примеси. Рассчитана взаимосвязь возбужденных состояний с атомным номером примеси.

Преимущество предложенного подхода и полученных результатов перед, например, расчетами в рамках метода сильной связи или на основе теории функционала плотности состоит в получении фактически аналитического выражения для огибающей, которое может быть легко использовано при расчетах времен переходов между основным и возбужденными состояниями примеси (последние сохраняют водородоподобную структуру).

\section{Благодарности}

Авторы признательны В.В. Цыпленкову и В.А. Бурдову за интерес к работе и стимулирующие обсуждения.

\section{Финансирование работы}

Работа поддержана грантом РНФ 19-72-20163.

\section{Конфликт интересов}

Авторы заявляют, что у них нет конфликта интересов.

\section{Список литературы}

[1] J.A. Burton. Physica, 20, 845 (1954).

[2] C. Kittel, A.H. Mitchell. Phys. Rev., 96, 1488 (1954).

[3] J.J. Pla, K.Y. Tan, J.P. Dehollain, W.H. Lim, J.J.L. Morton, D.N. Jamieson, A.S. Dzurak, A. Morello. Nature, 489, 541 (2012).

[4] G. Wolfowicz, A.M. Tyryshkin, R.E. George, H. Riemann, N.V. Abrosimov, P. Becker, H.-J. Pohl, M.L.W. Thewalt, S.A. Lyon, J.J.L. Morton. Nature Nanotechnol., 8, 561 (2013).

[5] H.-W. Hübers, S.G. Pavlov, H. Riemann, N.V. Abrosimov, R.Kh. Zhukavin, V.N. Shastin. Appl. Phys. Lett., 84, 3600 (2004). 
[6] S.G. Pavlov, N. Deßmann, V.N. Shastin, R.Kh. Zhukavin, B. Redlich, A.F.G. van der Meer, M. Mittendorff, S. Winnerl, N.V. Abrosimov, H. Riemann, H.-W. Hübers. Phys. Rev. X, 4, 021009 (2014).

[7] M. Fuechsle, J.A. Miwa, S. Mahapatra, H. Ryu, S. Lee, O. Warschkow, L.C.L. Hollenberg, G. Klimeck, M.Y. Simmons. Nature Nanotechnol., 7, 242 (2012).

[8] A.K. Ramdas, S. Rodriguez. Rep. Prog. Phys., 44, 1297 (1981).

[9] A.J. Sigillito, A.M. Tyryshkin, J.W. Beeman, E.E. Haller, K.M. Itoh, S.A. Lyon. Phys. Rev. B, 94, 125204 (2016).

[10] N. Deßmann, S.G. Pavlov, V.N. Shastin, R.Kh. Zhukavin, V.V. Tsyplenkov, S. Winnerl, M. Mittendorff, N.V. Abrosimov, H. Riemann, H.-W. Hübers. Phys. Rev. B, 89, 035205 (2014).

[11] H. Ye, J. Yu. Sci. Technol. Adv. Mater., 15, 024601 (2014).

[12] J.H. Reuszer, P. Fisher. Phys. Rev., 135, A1125 (1964).

[13] S.D. Seccombe, D.M. Korn. Solid State Commun., 11, 1539 (1972).

[14] R.A. Faulkner. Phys. Rev., 184, 713 (1969).

[15] H. Fritzsche. Phys. Rev., 120, 1120 (1960).

[16] A. Baldereschi. Phys. Rev. B, 1, 4673 (1970).

[17] S.T. Pantelides, C.T. Sah. Phys. Rev. B, 10, 621 (1974).

[18] Р.Х. Жукавин, К.А. Ковалевский, С.М. Сергеев, Ю.Ю. Чопорова, В.В. Герасимов, В.В. Цыпленков, Б.А. Князев, Н.В. Абросимов, С.Г. Павлов, В.Н. Шастин, Г. Шнайдер, Н. Дессманн, О.А. Шевченко, Н.А. Винокуров, Г.Н. Кулипанов, Г.-В. Хьюберс. Письма ЖЭТФ, 106, 555 (2017).

[19] Р.Х. Жукавин, К.А. Ковалевский, Ю.Ю. Чопорова, В.В. Цыпленков, В.В. Герасимов, П.А. Бушуйкин, Б.А. Князев, Н.В. Абросимов, С.Г. Павлов, Г.-В. Хьюберс, В.Н. Шастин. Письма ЖЭТФ, 110, 677 (2019).

[20] G. Pica, B.W. Lovett. Phys. Rev. B, 94, 205309 (2016).

[21] A.J. Sigillito, A.M. Tyryshkin, J.W. Beeman, E.E. Haller, K.M. Itoh, S.A. Lyon. Phys. Rev. B, 94, 125204 (2016).

[22] В.В. Цыпленков, В.Н. Шастин. ФТП, 52, 1469 (2018).

[23] S. Zwerdling, B. Lax, L.M. Roth, K.J. Button. Phys. Rev., 114, 80 (1959).

[24] W. Kohn, J.M. Luttinger. Phys. Rev., 98, 915 (1955).

[25] B.W. Levinger, D.R. Frankl. J. Phys. Chem. Solids, 20, 281 (1961).

[26] R. Resta. Phys. Rev. B, 16, 2718 (1977).

[27] G. Cappellini, R. Del Sole, L. Reining, F. Bechstedt. Phys. Rev. B, 47, 9892 (1993).

[28] J.P. Walter, M.L. Cohen. Phys. Rev. B, 2, 1821 (1970).

[29] V.A. Belyakov, V.A. Burdov. Phys. Rev. B, 79, 035302 (2009).

[30] S.T. Pantelides, C.T. Sah. Phys. Rev. B, 10, 638 (1974).

[31] L. Resca, R. Resta, H.B. Shore. Phys. Rev. B, 25, 4031 (1982).

[32] T.G. Castner, jr. Phys. Rev. B, 79, 195207 (2009).

\section{Electronic states of group $\mathbf{V}$ donors in germanium: variational calculation taking into account the short-range potential}

\author{
A.A. Revin ${ }^{1,2}$, A.M. Mikhaylova ${ }^{1,2}$, A.A. Konakov ${ }^{1,2}$, \\ V.N. Shastin ${ }^{2}$ \\ ${ }^{1}$ National Research Lobachevsky State University \\ of Nizhny Novgorod, \\ 603950 Nizhny Novgorod, Russia \\ ${ }^{2}$ Institute for Physics of Microstructures, \\ Russian Academy of Sciences, \\ 603087 Nizhny Novgorod, Russia
}

\begin{abstract}
In the framework of the envelope function approximation, the wave functions of low-lying $1 s\left(A_{1}\right), 2 s, 2 p_{0}, 2 p_{ \pm}$, $3 p_{0}$ states of shallow donor centers $\mathrm{P}, \mathrm{As}, \mathrm{Sb}$ in germanium are calculated considering the short-range part of the impurity potential. The latter is constructed individually for each impurity, taking into account the spatial dispersion of the dielectric function and the difference between the ionic cores of germanium and the impurity center. The envelope function equation was solved using the Ritz variational method, and selected trial wave functions of the orbitally non-degenerate $s$-states are characterized by two spatial scales: the first one is of the order of the donor effective Bohr radius and corresponds to the long-range part of the potential, and the second one, which is an order of magnitude less, simulates the electron response to the short-range part of the donor potential. The electron density in the donor ground state is shifted to the nucleus due to the attractive ,central cell“ correction. The envelope functions of $p$-states, in turn, are constructed in such a way they are orthogonal to the ground state envelope functions for each impurity center, and, unlike previous works, are different for various donors.
\end{abstract}

